\title{
Optimising Efficiency and Gain of Small Meander Line RFID Antennas using Ant Colony System
}

\author{
Andrew Lewis, Gerhard Weis, Marcus Randall, Amir Galehdar and David Thiel
}

\begin{abstract}
Radio Frequency IDentification (RFID) technology is increasingly being used to uniquely identify objects. An important component of RFID systems is the design of the antenna - which usually takes the form of a compacted meander line. This task becomes an optimisation problem as different designs will have different efficiencies and resonant frequencies. In this paper, we explore the use of a multi-objective version of ant colony system. This constructive meta-heuristic, as shown, is highly suitable for this problem.
\end{abstract}

Keywords: ant colony system optimisation, muti-objective optimisation, RFID antenna design.

\section{INTRODUCTION}

$\mathbf{T}$ HE automated design of meander line RFID (radio frequency identification) antennas is relatively new. Traditionally, design engineers would create these manually in a time-consuming, iterative process. However, this is only practicable for small and relatively simple antennas. Recent work has shown that heuristic search techniques are capable of producing very efficient antenna designs for larger problems [23], [28]. This paper extends that work by considering the simultaneous optimisation of two objectives, namely maximising antenna efficiency and minimising resonant frequency.

One approach to design of the general meander line antenna is to confine the antenna to a Cartesian grid. For a particular grid defining the meander line, a number of design choices are possible. By its very nature, this is a constructive task, as grid points are successively connected. Ant colony system (ACS) is a constructive meta-heuristic search algorithm and is one of the family of ant colony optimisation (ACO) techniques. Generating a meander line on a grid is a combinatorial problem that is a restricted and constrained form of the travelling salesman problem (TSP) - hence it is $\mathcal{N} \mathcal{P}$ hard [23]. There is a vast amount of literature covering ACO for the TSP (see Dorigo and Stützle [8] for an overiew). However, its use for antenna design has been limited to self structuring antennas [3] rather than the application presented in this paper.

The remainder of this paper is organised as follows. Section II gives a brief summary of RFID and meander line antennas while Section III describes ACS in general and how it is applied to this problem for the single objective case. Section IV extends this to show that ACS can effectively optimise

Andrew Lewis and Gerhard Weis are with the Institute for Integrated and Intelligent Systems, Griffith University, Queensland, Australia (email: \{gerhard.weis@student., a.lewis@\}griffith.edu.au). Marcus Randall is with the School of Information Technology, Bond University, Queensland, Australia (email: mrandall@bond.edu.au). Amir Galehdar and David Thiel are with the Centre for Wireless Monitoring and Applications, Griffith University, Queensland, Australia (email: \{s2145033@student., d.thiel@\}griffith.edu.au). both efficiency and resonant frequency. The computational results of the new system are given in Section V and finally, the conclusions and future research directions are presented in Section VI.

\section{RFID ANTENNAS}

The idea of RFID was first developed in 1948 [26]. Recently there have been many enhancements to this basic idea and the concept has found applications in many areas. One suggestion is the replacement of the universal price code (UPC) barcode system. Practical considerations have driven a need for smaller tags with longer reading range. The read range can be defined as "the maximum distance at which an RFID reader can detect the backscattered signal from the tag" [24]. This vital factor can be increased by designing antennas with higher gain which is directly related to the antenna efficiency. Design engineers seek the smallest, most efficient antenna structures for their RFID tags.

As mentioned above, one of the key features of RFID tag is antenna design. Traditionally, design engineers predict the performance of the antenna based on electromagnetic calculations which approximate real antenna performance. More recently, computers and accurate numerical modelling software applications have become available to explore antenna performance before construction. These codes are improved using mathematical optimisation techniques to produce the best possible solution. Many different optimisation techniques for antenna design are reported in the Special Issue of IEEE Transactions on Antennas and Propagation vol. 55 (3), March 2007. Meander line antennas are a subset of particular interest. An RFID system consists of two components:

- A transponder or tag - this has a small microchip and an antenna.

- A reader or scanner - this communicates with the tag and a host system which has the information of the identified tag item.

RFID tags are categorised into active and passive tags. Active tags have their own power source and passive tags harvest their's from the reader signal. Usually active and passive tags use different operating frequencies. Passive tags operating up to $100 \mathrm{MHz}$ work in the near field of the reader which means the range can be extended using lower frequencies. Since it is a near field operation, there are limits to the reading range.

Higher frequency tags operate beyond the near field of the reader and hence a longer reading can be achieved. Such tags use radio frequency backscatter which means the tag changes 
the antenna impedance by reflecting back some of the incident RF energy to the reader.

The antenna is the most crucial element for an RFID tag, because it is the power source, which makes good antenna gain essential in achieving a large transceiver range [15]. Other essential properties for an RFID antenna are size, robustness and manufacturing costs. The read range is directly proportional to the wavelength used. Lower frequencies or longer wavelengths tend to increase the read range, but also need larger antennas. The design goals for an RFID antenna is to make it small, efficient and with long reading distance.

Meander line antennas [27] are folded dipole antennas which increase radiation resistance without decreasing antenna bandwidth. They offer good radiation efficiency with considerable size reduction compared to conventional half or quarter wavelength antennas. Meander line antennas are of particular interest for RFID, due to their compactness and low manufacturing cost.

There is no generally applicable analytic design methodology for meander line antennas. So there is a need for computational methods for design optimisation. There have been some approaches using genetic algorithms to optimise RFID antennas [16], [20], however, they have concentrated on simple serpentine configurations or on very specific application areas. To date there have been little or no investigations of general configurations or computational optimisation has not been applied [9], [10].

\section{Ant Colony System}

Ant Colony Optimisation [6] is family of population optimisation paradigm encompassing a range of meta-heuristics based on the evolutionary mechanics of natural ant colonies. One of these variants is named Ant Colony System. These techniques have been applied extensively to benchmark problems such as the travelling salesman problem, the job sequencing problem and the quadratic assignment problem. Work on more complex problems, that have difficult constraints in such areas as transportation and telecommunications, has also been undertaken [6]. Like genetic algorithms, populations of solutions evolve over time. The major difference is that ACO represents a set of constructive techniques, i.e., each ant at each step of the generalised algorithm adds a component (such as the next city for the TSP) to its solution. Using simulated chemical or pheromone markers (conventionally denoted as $\tau$ ) as a collective form of self adaptation, populations produce increasingly better solutions.

The general operation of ant colony system may be described thus. Initially, a population of empty solutions is created. Each solution is referred to as an ant. The colony of ants proceeds to create solutions to a given problem by progressively adding solution component values (such as cities for the travelling salesman problem, or segments to neighbouring grid points in this problem) until each ant's solution is complete. The way that solution component values for ACS are selected is by the use of the pseudo-proportional rule [7]. Every time a solution component value is added, pheromone evaporation takes place to discourage other members to strictly follow another ant's path. This is referred to as local pheromone updating. At the end of this iteration of construction, the best solution is rewarded by having the pheromone levels associated with its solution components increased, while others are decayed. Over a number of such iterations, the quality of solutions will increase, until stagnation occurs.

The above description assumes only one objective function is to be optimised. The application described in this paper, and many other real-world tasks, requires the use of more than one objective. There has been much research undertaken to extend ACO metaphor to multi-objective optimisation. A brief summary of these researches follows.

Garcia-Martinez, Cordon and Herrera [12] compare a number of published multi-objective ant colony optimisation (MOACO) methods on a single set of bi-criteria TSPs. These methods are from Mariano and Morales [19], Iredi, Merkle and Middendorf [14], Gambardella, Taillard and Agazzi [11] and Doerner, Gutjahr, Hartl and Strauss [5]. For the first of these (named MOAQ), families of ants are used to optimise each objective. Each ant of the family learns from its same number in the preceding family. Additionally, infeasible solutions are penalised. Iredi et al. [14] propose two methods they entitle BicriterionAnt and BicriterionMC. Both use different pheromone repositories for the two objective functions. The main difference is that the former allows its ants to update both repositories while the latter will update one or the other (at each step of an iteration). Doerner et al. [5] use a variation on the idea of separate colonies in a scheme called COMPETants. After each iteration of the algorithm, the better performing colony gets a greater number of ants for the next generation. In contrast, the multiple ant colony system of Gambardella et al [11] uses a single pheromone matrix for the each colony, but uses multiple visibility heuristics.

Population ACO (PACO $)^{1}$ has also been used to solve multiple objective optimisation problems [13], [1]. Rather than keeping separate colonies of ants for each objective function, PACO creates different sub-populations to update each of the pheromone matrices (one for each objective). A variation of this is Crowding PACO (CPACO) [1] in which a population, without sub-populations, is maintained. Each solution of the current population of ants is compared (in terms of solution quality) to a subset of the population. Given that two solutions are approximately similar, the old population member will be replaced if the other dominates it. Using a set of bi-criteria TSPs, it was shown that that CPACO was able to outperform PACO.

\section{A. Ant Colony System Applied to Meander Line Antennas with a Single Objective}

The canonical ACS algorithm needs to be adapted for the problem of creating meander line antennas [23]. That study and the work by Weis et al. [28] only consider efficiency as

\footnotetext{
${ }^{1} \mathrm{PACO}$ uses a population of solutions to derive its pheromone matrix, rather than directly from the solution components.
} 
the objective. Figure 1 (a) shows a $5 \times 5$ grid on which the ACS algorithm operates and (b) a possible solution. The ACS algorithm only solves one half of the antenna. The solution is then mirrored on the other half of the antenna, with a connecting $1 \mathrm{~mm}$ bridge. For the antennas used in this paper (resonating at $433 \mathrm{MHz}$ ), each half area, or grid, contains $n \times n$ points. The physical size of the grid remains constant at $25 \mathrm{~mm}$, despite different values of $n$. Each ant attempts to construct a meander line that begins on a boundary point and traverses all points on the grid.

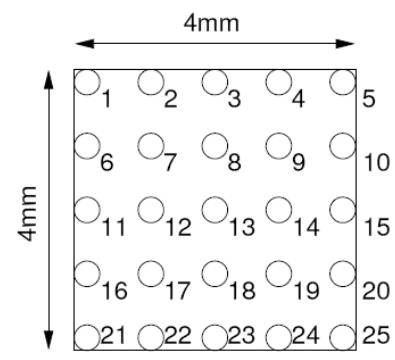

(a)

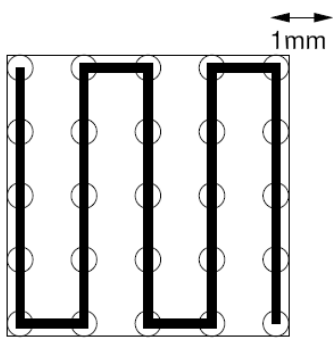

(b)
Fig. 1. (a) defines the grid and numbering system and (b) shows a possible meander line antenna. The latter is referred to as a "plough" structure.

The difficulty for the algorithm in constructing Hamiltonian walks (as reported by Randall et al. [23]) is that each point is only connected to at most four others. It is quite likely that an ant will "trap" itself within the grid, as it has already visited all the neighbouring points.

To minimise the potential of an ant becoming stuck, the ACS algorithm for meander line antennas works in the following way. Each ant starts at a random chosen boundary point and walks to the next chosen neighbour inside the boundaries that are not already visited. The ants are guided by the following three factors:

1) Pheromone - The pheromone matrix is an $n^{2} \times 4$ structure. The latter dimension is direction. The pheromone component of the characteristic pseudo-proportional equations is given as $\tau(c, d)$ where $c$ is the current grid position and $d$ is the direction.

2) Lookahead function - In order to move each ant, the number of unused neighbours of each of the neighbours of the current point is calculated. The greater this number, the higher the probability of choosing that direction.

3) Straight line segment function - Encouraging straight line segments helps to ensure that all $n^{2}$ points are visited by the meander line. This is easily calculated by comparing the current candidate direction with the previous direction that the ant has taken.

If the number of unused neighbours for an ant is 0 , then the ant has become stuck and is considered dead (if not all points are visited by this ant). Dead ants are disregarded for the remainder of the iteration. At the end of each iteration, all ants that have valid solutions have these evaluated by the NEC [2] antenna suite. Therefore, the calculation of the efficiency of the generated antennas is a black box to this algorithm, and the ants can not use any incremental objective information as guidance.

Another important issue in the automated design of meander line antennas is that of structure refinement or local search to a completed solution. ACS does not guarantee that an ant's solution will be locally optimal. Traditional ACS implementations use standard operators such as move and swap in order to find better solutions - using an original ant solution as a base. The difficulty in this application, as previously mentioned, is that the objectives (efficiency and resonant frequency) are black boxes. Thus, no guiding information is available. The work by Weis et al [28] showed that the application of the backbite operator [18], [25] could dramatically increase solution quality. The operator manipulates the end of the antenna, changing the configuration slightly, but ensuring another valid antennas is produced. Additionally, Weis et al. [28] extends the metaphor so that a hierarchical group of backbite operators could be performed that would cover all backbite configuration possibilities. The depth of the search tree was set to three. This ensured a good exploration of local space surrounding an antenna, without overusing computational resources.

\section{A Multiobjective Model}

Previous work by the authors [23], [28] has demonstrated that heuristic search algorithms in general, and ACS in particular, can be used very effectively to improve the efficiency of small, meander line RFID antennas. In most realworld applications, however, an "optimal" solution involves simultaneously satisfying several objectives. Another issue of importance in the design of these antennas is that of the resonant frequency of operation. High Frequency (HF) RFID, which operates at $13.56 \mathrm{MHz}$, has been widely deployed for item management applications but fails where read distances of greater than $1 \mathrm{~m}$ are required. More recently, Ultra High Frequency (UHF) has been investigated, providing for smaller antennas and longer read distances. UHF RFID has been developed to operate at a number of different frequencies, specifically, $433 \mathrm{MHz}, 860-956 \mathrm{MHz}$ and $2.45 \mathrm{GHz}$. In this paper, we consider antennas in the $433 \mathrm{MHz}$ band, as they are commonly used in real-world applications.

It should be noted that evaluation is just a part, perhaps the lesser part, of the optimisation task. Designing a candidate antenna structure is a significant part of the problem, for which the constructive nature of the ACO path planning method is particularly appropriate. Attempting to use another optimisation algorithm, such as a Genetic Algorithm (GA) or Particle Swarm Optimisation (PSO) still leaves the antenna designer with the problem of how to construct a feasible structure for the antenna in order to evaluate it. In addition, the local permutation operations of such algorithms are highly likely to overwhelmingly generate infeasible solutions, even given a feasible starting structure. Because of these difficulties, there is very little reported in the literature on the application of optimisation algorithms to the automated design of meander line antennas, with the exception of some work on 
the optimisation of specific, limited forms, such as serpentine antennas [21].

For the antenna design problem being considered maximising the antenna efficiency, $\eta$, and minimising the resonant frequency, $f_{0}$, are the two design objectives. In order to be able to process these objectives the ACO algorithm had to be modified. When optimising for a single design objective, it is simple to determine which ant has achieved the best solution, and should thus be allowed to globally update the pheromone matrix; it is the ant whose solution has the best value for the objective. However, when more than one objective is involved, the question of what is the "best" solution can be difficult to answer, particularly if objectives conflict. What is ideally required in this case is a method that delivers information on the trade-off between objectives.

Our multi-objective implementation is simpler and requires fewer computational resources than those papers outlined in the previous section, as only one ant colony is used. To determine whether one solution is more attractive than another, a domination relation is used. For solution vectors $\overrightarrow{x_{1}}$ and $\overrightarrow{x_{2}}$, when the following conditions are met:

- $\overrightarrow{x_{1}}$ is at least as good as $\overrightarrow{x_{2}}$ for all the objectives, and

- $\overrightarrow{x_{1}}$ is strictly better than $\overrightarrow{x_{2}}$ for at least one objective

then $\overrightarrow{x_{1}}$ is said to "dominate" $\overrightarrow{x_{2}}$ (denoted $\overrightarrow{x_{1}} \prec \overrightarrow{x_{2}}$ ). In the case where $\overrightarrow{x_{1}}$ and $\overrightarrow{x_{2}}$ dominate other solution vectors but not each other they are deemed mutually optimal solutions and referred to as Pareto-optimal. The set of Pareto-optimal solutions reflects the trade-off surfaces between the different objectives and is kept in a data structure referred to as the archive. This set of Pareto-optimal solutions is referred to as the Pareto-front.

This approach to the optimisation of multiple objectives delivers not just a single solution but a set of (Pareto-optimal) solutions. Design engineers must still make some decision as to which particular solution is most fit for the requirements of a specific application. To do this they must choose between Pareto-optimal solutions based on some set of preferences ranking the different objectives. This choice can be generally be made in one of three ways [4]:

- a priori - a set of weights is determined for the different objectives and they are aggregated, usually by a simple algebraic sum. This aggregated objective function is then used to drive a single-objective optimisation algorithm. It is well known, however, that this approach commonly experiences difficulties finding solutions on Pareto-fronts of particular shapes [17] and can thus fail to deliver adequate solutions.

- progressive - the designer interactively supplies information about design preferences as the optimisation progresses. This is well-suited to problems where the objectives cannot easily be expressed in simple, numerical terms but tends to be laborious and time-consuming.

- a posteriori - the optimisation algorithm makes no attempt at ranking Pareto-optimal solutions but delivers the whole set to the designer for a decision to be made $\overline{\text { Algorithm } 1 \text { A single iteration of the multi-objective ACS }}$ algorithm.

1: A population of ants begin construction of the walks (antennas) on a Cartesian grid of specified size

2: while each ant has not completed construction of an antenna do

3: for each ant, from a starting node on the edge of the grid do

4: Add next node to a directed graph, according to the probabilistic selection rules outlined in Section III, coupled with next neighbour lookahead and straight line weighting, if appropriate

5: If the ant cannot find a feasible next node, terminate the ant for this iteration

6: end for

7: $\quad$ Update local pheromone data for all ants

8: end while

9: Apply the backbite operator to degree three to each solution/antenna

10: Determine if any of the population of solutions should be added to the Archive

11: For those solutions entering the archive, update the global pheromone

12: end

after the algorithm terminates. This can provide insight into the behaviour of systems in response to design parameters in addition to delivering particular solutions. The experiments described in this work use this approach.

Antenna structures constructed by the ants, and refined using the backbite operator [28], are now evaluated by the NEC software and two objective values returned, for $\eta$ and $f_{0}$. The Pareto dominance relationships between different solutions are determined, and Pareto-optimal solutions accumulated in a continuously-updated archive. In the modified ACS, all ants that deliver Pareto-optimal solutions at an iteration are allowed to contribute an update to the pheromone matrix, the amount of their update being inversely proportional to the number of contributing ants. This is slightly different to the PACO approach where the whole population is used to derive the pheromone matrix.

Algorithm 1 gives an overall mechanical description of the multi-objective ACS implementation.

\section{COMPUtATIONAL ExPERIMENTS AND RESUlts}

As in Randall et al. [23] and Weis et al. [28] meander lines on grids ranging from $5 \times 5$ to $10 \times 10$, running the ACS algorithm ten times (by varying the random initial seed used for the probabilistic operations of the algorithm) are solved. Each search was permitted 1000 iterations. The structures obtained by the ants were refined using the backbite operator, with a fixed tree depth of three. An archive was maintained of all Pareto-optimal solutions obtained for each run. Thus, the cache of previously-computed results was converted to a persistent 
database reusable across experimental runs, to further reduce the computational cost of performing the experiments.

In contrast to the previous works [23], [28], the relaxation of the requirement that antenna elements be Hamiltonian walks on the grid meant every ant was able to produce a "feasible" solution requiring evaluation by the NEC software in this study. The previous restriction was aimed at maximising the antenna length, in order to implicitly minimise the resonant frequency since lower frequencies tend to increase the antenna read range, but also need larger antennas. As resonant frequency was now an explicit objective of the optimisation process, its "optimisation" via artificial constraints was no longer necessary. With this relaxation, there was a subsequent increase in the number of feasible solutions to be evaluated. Thus the use of parallel computing resources became a practical necessity. An ad hoc computational grid was formed from a pool of about 20 computers, a mix of Intel P4 and Athlon X64 dual-core-based machines. The computers used were not dedicated to this task; jobs were distributed to machines according to their instantaneous ability to process them, taking into account machine load from other sources. For some experimental runs a number of computers may not have been available at all. The dual-core computers were always issued two jobs, when they were used.

Our first experiments explored the effect of greediness in the selection of the next solution component value an ant takes on. The pseudo-proportional rule (as discussed in Section III) consists of two branches; an ant may choose a value based on greedy selection or a value chosen probabilistically (according to the value's fitness). The ACS parameter, $q_{0}$, governs this choice. Note that $0 \leq q_{0} \leq 1$. A high value of $q_{0}$ leads to more greedy choices being made and vice versa. Experiments for different values of $q_{0}$ at each grid size were processed sequentially, due to the limited computing resources available. In Table I the difference between the number of structure evaluations requested and the actual number computed is attributable to the effect of caching the results; it may be seen at each grid size that the number of unique solutions that need be evaluated progressively decreases as the persistent cache fills with each subsequent run. Very noticeable for the smaller grid sizes, this effect diminishes as grid size increases and the total search space the algorithm explores grows exponentially. It may also be noticed this effect remains significant with greater values of $q_{0}$ : as the algorithm becomes "greedier" it tends to attempt to reuse paths already investigated. The computational results of the experiments are given in Table I. By inspection of the table, it may be noted that up to 68,372 structures were computed, for one of the $10 \times 10$ runs. That would have taken over 112,000 seconds ( 31 hours) had these all been computed sequentially. The elapsed time for the run was only 13,926 seconds (about 4 hours), a significant improvement.

Table I also reports structure evaluation times much lower than those reported in the previous studies [23], [28]. This is the result of a combination of two main factors:

- Relaxing the requirement that antenna paths be Hamil-
TABLE I

Computational Results For antennas In the 433 MHz Band. AlL TIMES GIVEN IN SECONDS. AVERAGES ARE PER PROCESSOR CORE.

\begin{tabular}{ccccccc}
\hline $\begin{array}{c}\text { Grid } \\
\text { Size }\end{array}$ & $q_{0}$ & $\begin{array}{c}\text { Average CPU } \\
\text { time per } \\
\text { structure }\end{array}$ & $\begin{array}{c}\text { Elapsed } \\
\text { time }\end{array}$ & $\begin{array}{c}\text { Average } \\
\text { structures } \\
\text { computed }\end{array}$ & $\begin{array}{c}\text { Total } \\
\text { structures } \\
\text { computed }\end{array}$ & $\begin{array}{c}\text { Total } \\
\text { structures } \\
\text { requested }\end{array}$ \\
\hline 5 & 0.1 & 0.58 & 1290 & 373 & 2394 & 20286 \\
5 & 0.5 & 0.54 & 1296 & 213 & 2244 & 17248 \\
5 & 0.9 & 0.55 & 498 & 54 & 534 & 12128 \\
\hline 6 & 0.1 & 0.41 & 4673 & 712 & 13540 & 39420 \\
6 & 0.5 & 0.59 & 3444 & 376 & 8573 & 25856 \\
6 & 0.9 & 0.71 & 1711 & 183 & 3009 & 15190 \\
\hline 7 & 0.1 & 0.86 & 14097 & 5856 & 44064 & 59522 \\
7 & 0.5 & 0.87 & 5546 & 734 & 17900 & 38650 \\
7 & 0.9 & 0.96 & 1711 & 202 & 3009 & 15190 \\
\hline 8 & 0.1 & 1.06 & 9658 & 2737 & 56161 & 63620 \\
8 & 0.5 & 1.31 & 7885 & 1566 & 37387 & 50267 \\
8 & 0.9 & 1.48 & 5150 & 580 & 13223 & 29743 \\
\hline 9 & 0.1 & 1.34 & 17533 & 1885 & 61596 & 64200 \\
9 & 0.5 & 1.53 & 10215 & 1846 & 48966 & 55585 \\
9 & 0.9 & 1.59 & 7313 & 971 & 23688 & 32497 \\
\hline 10 & 0.1 & 1.65 & 13926 & 2535 & 68372 & 69268 \\
10 & 0.5 & 1.76 & 21140 & 2373 & 64379 & 65925 \\
10 & 0.9 & 2.21 & 7313 & 1350 & 23688 & 32497 \\
\hline
\end{tabular}

tonian leads to a reduction in the average length and complexity that must be simulated by the NEC software, which reduces the computation and time required.

- With the anticipated increase in the number of evaluations to be performed, the evaluation procedure was altered to reduce the computational cost. In the earlier experiments resonant frequency was determined by sweeping the operating frequency across a band of interest, evaluating antenna performance at a large number of discrete frequencies. This was modified to use a binary search algorithm within the same band, greatly reducing the number of discrete frequencies test ed, and the time taken.

To design an UHF antenna able to operate at a resonant frequency in the $433 \mathrm{MHz}$ band (commonly used in practical applications), suitable construction parameters were: a track width of $1 \mathrm{~mm}$, an antenna half-area to $25 \times 25 \mathrm{~mm}^{2}$ and the bridge for the feed-point between the dipole arms of $6 \mathrm{~mm}$.

Figure 2 shows the $\eta f_{0}$ attainment surface obtained for all grid densities. The resonant frequency range was $350 \mathrm{MHz}$ $<f_{0}<1520 \mathrm{MHz}$ and efficiency range was $70.7 \%<\eta<$ $99.8 \%$. The standard operating frequency in this band is 433 MHz. From the figure it is evident that optimised antenna structures have been obtained capable of operating at this frequency.

Some measure of the quality of the attainment surface can be inferred from the fact it includes a solution found, by exhaustive enumeration, to be the globally best solution, in terms of gain [9] for one of the grid sizes. It is evident from consideration of the number of feasible solutions for larger grid sizes (over $10^{13}$ for grids of $10 \times 10$ ) that similar validation for the whole of the attainment surface is difficult to provide.

Figure 3 shows the structure of the antenna element with resonant frequency closest to this desired operating frequency $\left(f_{0}=430 \mathrm{MHz}, \eta=83.6 \%\right)$. This antenna was constructed using a grid density of $10 \times 10$; inspection of Pareto-fronts for other grid densities showed feasible structures could also 
TABLE II

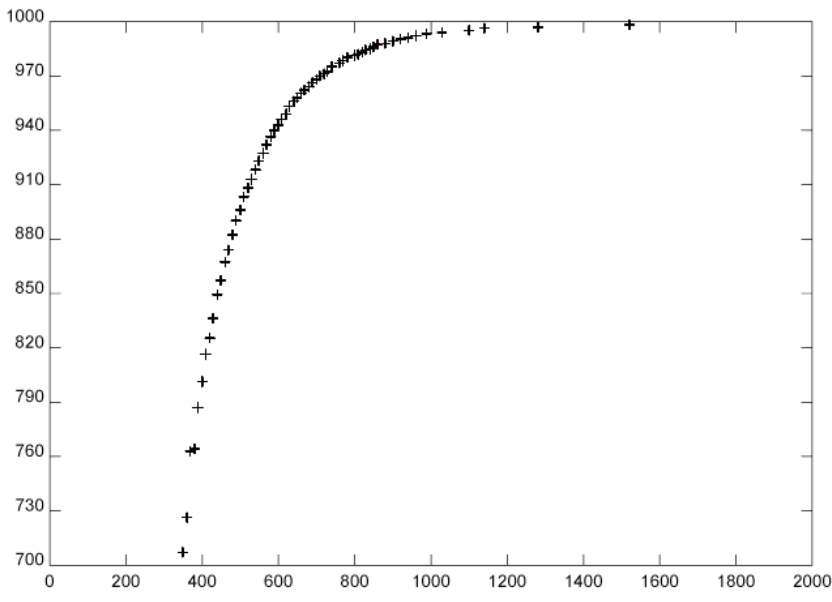

Performance of ANTENNAS IN THE 433MHz UHF BAND.

\begin{tabular}{ccc}
\hline $\begin{array}{c}\text { Grid } \\
\text { Size }\end{array}$ & $\begin{array}{c}\text { Resonant } \\
\text { Frequency } \\
\text { (MHz) }\end{array}$ & $\begin{array}{c}\text { Maximum } \\
\text { Efficiency\% }\end{array}$ \\
\hline 5 & 570 & 92.8 \\
6 & 510 & 89.5 \\
7 & 460 & 85.7 \\
8 & 430 & 82.8 \\
9 & 430 & 83.5 \\
10 & 430 & 83.6 \\
\hline
\end{tabular}

Fig. 2. $\eta f_{0}$ attainment surface for the $25 \times 25 \mathrm{~mm}^{2}$ antenna.

be obtained using grid densities of $9 \times 9$ and $8 \times 8$ but that the minimum resonant frequency obtainable with $7 \times 7$ was $460 \mathrm{MHz}$ and lower grid densities had correspondingly higher minimum resonant frequencies. These results are summarised in Table II. The resolution of the resonant frequency was 10 $\mathrm{MHz}$.

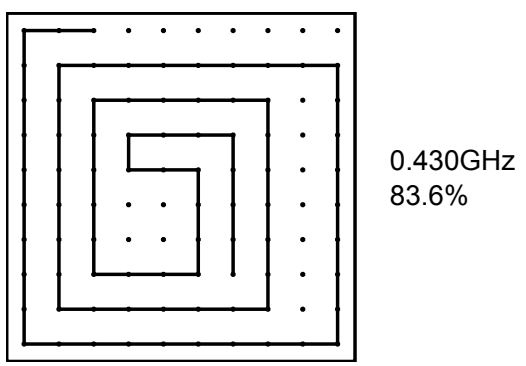

Fig. 3. Structure of the $10 \times 10$ antenna element with resonant frequency, $f_{0}=430 \mathrm{MHz}$

\section{CONCLUSIONS}

The experiments described in this paper have demonstrated that the combination of ACO with the NEC antenna analysis software is a practical and effective means of designing electrically small, meander line, RFID antennas to meet realworld design criteria. Antenna structures capable of operating with high efficiency at standard frequencies were found rapidly by an automatic process, an engineer only being required to choose a specific antenna design from several Pareto-optimal alternatives presented, according to preference.

For a standard, $433 \mathrm{MHz}$ antenna designed to fit on a $25 \times$ $56 \mathrm{~mm}$ substrate, the longest elapsed time for a single ACO run was a little under 6 hours, despite being constrained to use of a reasonably small number of heterogeneous, commodity

computing resources of highly dynamic availability. While to complete the experiment described took a further 17 runs (of times ranging from under 10 minutes to a few hours) with sufficient computers available the entire investigation could have been completed in this 6 hour period, as each ACS run was independent of all others and could be performed concurrently.

Beyond this, we wish to investigate other constructive heuristics with which to build meander lines as a basis of comparison to ACS. In addition, it may be interesting to investigate other local search operators suited to this problem and $\mathrm{ACO}$, such as multi-objective $\mathrm{A}^{*}$ [22], and their impact on the convergence behaviour of the algorithm.

\section{REFERENCES}

[1] D. Angus. Crowding population-based ant colony optimisation for the multi-objective travelling salesman problem. In $2^{\text {nd }}$ IEEE International $e$-Science and Grid Computing Conference. IEEE, 2006. (Workshop on Biologically-inspired Optimisation Methods for Parallel and Distributed Architectures: Algorithms, Systems and Applications).

[2] G. Burke, A. Poggio, J. Logan, and J. Rockway. NEC - Numerical electromagnetics code for antennas and scattering. Antennas and Propagation Society International Symposium, 17:147-150, June 1979.

[3] C. Coleman, E. Rothwell, and J. Ross. Investigation of simulated annealing, ant-colony optimization, and genetic algorithms for selfstructuring antennas. Antennas and Propagation, IEEE Transactions on, 52(4):1007-1014, 2004.

[4] Y. Collette and P. Siarry. Multiobjective Optimization. Springer-Verlag, 2003.

[5] K. Doerner, W. Gutjahr, R. Hartl, and C. Strauss. Pareto ant colony optimization: A metaheuristic approach to multiobjective portfolio selection. Annals of Operations Research, 131:79-99, 2004.

[6] M. Dorigo and G. Di Caro. The ant colony optimization metaheuristic. In D. Corne, M. Dorigo, and F. Glover, editors, New Ideas in Optimization, pages 11-32. McGraw-Hill, London, 1999.

[7] M. Dorigo and L. Gambardella. Ant Colony System: A cooperative learning approach to the traveling salesman problem. IEEE Transactions on Evolutionary Computation, 1(1):53-66, 1997.

[8] M. Dorigo and T. Stützle. Ant Colony Optimization. MIT Press, 2004.

[9] A. Galehdar, D. Thiel, and S. O'Keefe. Antenna efficiency calculations for electrically small, RFID antennas. IEEE Antenna and Wireless Propagation Letters, 6:156-159, 2007.

[10] A. Galehdar, D. Thiel, S. O'Keefe, and S. Lingsley. Efficiency variations in electrically small, meander line RFID antennas. In Proceedings of IEEE Antenna Propagation Symposium, 2007.

[11] L. Gambardella, E. Taillard, and G. Agazzi. MACS-VRPTW - A multiple ant colony system for vehicle routing pr oblems with time windows. In D. Corne, M. Dorigo, and F. Glover, editors, New Ideas in Optimization, pages 63-76. McGraw-Hill, London, 1999.

[12] C. Garcia-Martinez, O. Cordon, and F. Herrera. A taxonomy and empirical analysis of multiple objective ant colony optimization algorithms for bi-criteria TSP. European Journal of Operational Research, 180:116148,2007 
[13] M. Guntsch and M. Middendorf. Applying population based ACO to dynamic optimization problems. In M. Dorigo, G. Di Caro, and M. Sampels, editors, Third International Workshop on Ant Algorithms, ANTS 2002, volume 2463 of Lecture Notes in Computer Science, pages 111-122. Springer-Verlag, Brussels, Belgium, 2002.

[14] S. Iredi, D. Merkle, and M. Middendorf. Bi-criterion optimization with multi colony ant algorithms. In First International Conference on Evolutionary Multi-Criterion Optimization, volume 1993 of Lecture Notes in Computer Science, pages 359-372, Zurich, 2001. Springer Verlag.

[15] M. Keskilammi, L. Sydänheimo, and M. Kivikoski. Radio frequency technology for automated manufacturing and logistics control. Part 1: Passive RFID systems and the effects of antenna parameters on operational distance. The International Journal of Advanced Manufacturing Technology, 21(10):769-774, 2003.

[16] G. Kim and Y. Chung. Optimization of UHF RFID tag antennas using a genetic algorithm. IEEE Antennas and Propagation Society International Symposium, pages 2087-2090, July 2006.

[17] J. Koski. Defectiveness of weighting method in multicriterion optimization of structures. Communications in Applied Numerical Methods, 1:333-337, 1985

[18] M. Mansfield. Monte Carlo studies of polymer chain dimensions in the melt. The Journal of Chemical Physics, 77(3):1554-1559, 1982.

[19] C. Mariano and E. Morales. A multiple objective Ant-Q algorithm for the design of water distribution irrigation networks. Technical Report HC-9904, Instituto Mexicano de Tecnologia del Agua, June 1999.

[20] G. Marrocco. Gain-optimized self-resonant meander line antennas for
RFID applications. Antennas and Wireless Propagation Letters, 2:302305, 2003.

[21] G. Marrocco, A. Fonte, and F. Bardatti. Evolutionary design of miniaturized meander-line antennas for RFID applications. In Antennas and Propagation Society International Symposium, 2002, volume 2, pages 362-365. IEEE, 2002.

[22] M. Ngugen, S. Alam, and H. Abbass. Dynamic weather avoidance in a traffic constrained airspace. In Proceedings of the 6th Eurocontrol Innovation Research Workshop and Conference, 2007.

[23] M. Randall, A. Lewis, A. Galehdar, and D. Thiel. Using ant colony optimisation to improve the efficiency of small meander line RFID antennas. In $3^{\text {rd }}$ IEEE International e-Science and Grid Computing Conference, 2007.

[24] K. Seshagiri, R. Nikitin, and S. Lam. Antenna design for UHF RFID tags: A review and a practical application. IEEE Transactions on Antennas Propagation, 53:3870-3876, 2005.

[25] A. Sokal. Monte carlo methods for the self avoiding walk. Monte Carlo and Molecular Dynamics Simulations in Polymer Science, pages 47-124, March 1994.

[26] H. Stockman. Communication by means of reflected power. Proceedings of the Institute of Radio Engineers, pages 1196-1204, 1948.

[27] T. Warnagiris and T. Minardo. Performance of a meandered line as an electrically small transmitting antenna. Antennas and Propagation, IEEE Transactions on, 46(12):1797-1801, 1998.

[28] G. Weis, A. Lewis, M. Randall, A. Galehdar, and D. Thiel. Local search for ant colony system to improve the efficiency of small meander line rfid antennas. In Proceedings of the Congress on Evolutionary Computation, pages $1708-1713,2008$. 